
on Metabolomics

\title{
Profiling Redox and Energy Coenzymes in Tissue and Blood using NMR Spectroscopy
}

G. A. Nagana Gowda ${ }^{1,2 *}$, Lauren Abell ${ }^{2}$, Chi Fung Lee ${ }^{2}$, Rong Tian ${ }^{2}$ and Daniel Raftery ${ }^{1,2 *}$

${ }^{1}$ Northwest Metabolomics Research Center and ${ }^{2}$ Mitochondria and Metabolism Center Anesthesiology and Pain Medicine, University of Washington, USA 


\section{Profiling Redox and Energy Coenzymes in Tissue and Blood using NMR Spectroscopy}

\section{Graphical Abstract}

Whole Blood

Cardiac Tissue

Liver Tissue

Brain Tissue

Skeletal muscle tissue

Kidney Tissue

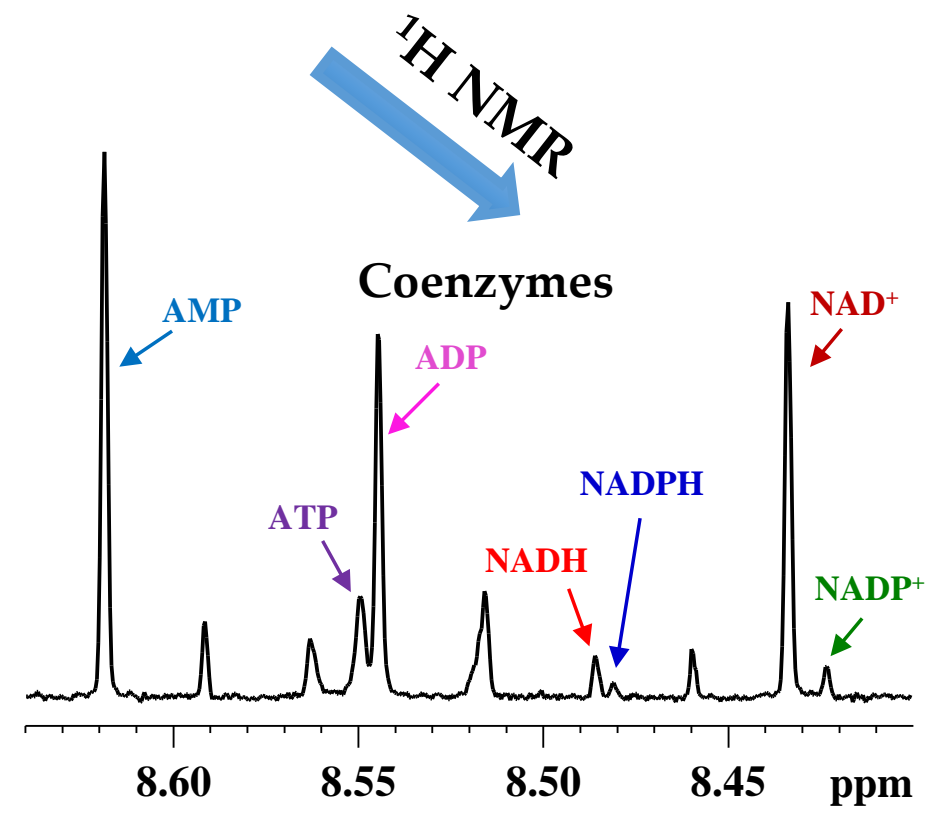

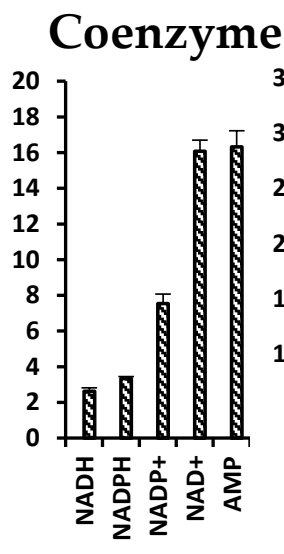

Antioxidants

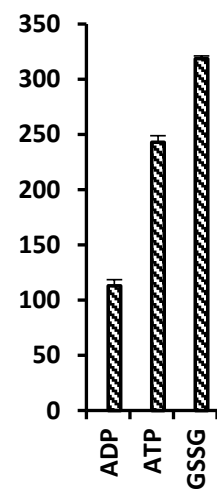

$\left.\begin{array}{r}700 \\ 600 \\ 500 \\ 400 \\ 300 \\ 200 \\ 100 \\ 0\end{array}\right]$

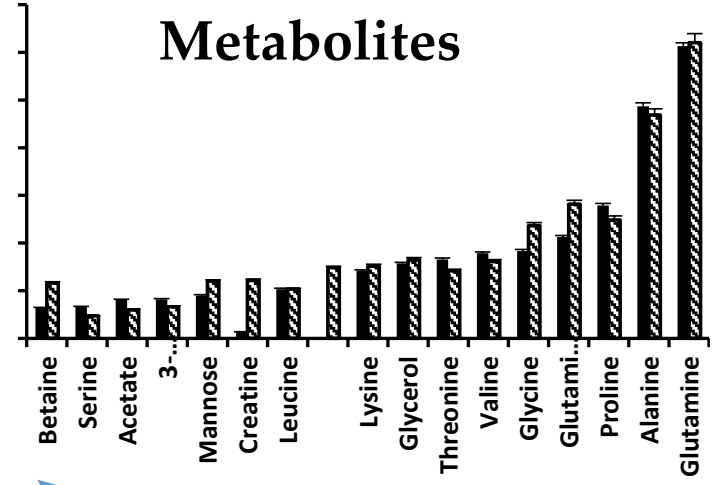

GSSG

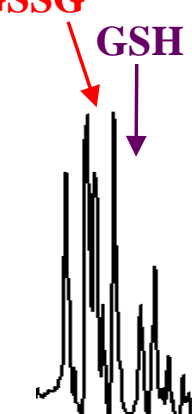

GSH

Antioxidants

GSH GSSG

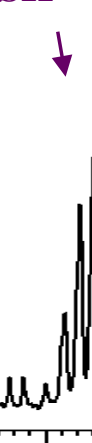

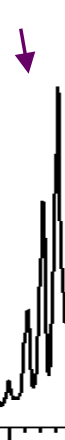

2.9

$2.8 \quad 2.7$

ppm

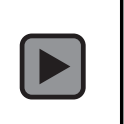


Abstract: Coenzymes of redox reactions and cellular energy mediate biochemical reactions fundamental to the functioning of all living cells. To date, no simple method exists to profile these coenzymes in biological samples such as blood and different types of tissue in a single step. Conventional methods that measure them individually carry high risk of errors outweighing their biological variations. We show here that coenzymes including antioxidants and a vast pool of other metabolites can be profiled quantitatively using a simple ${ }^{1} \mathrm{H}$ NMR experiment, simultaneously, in tissue and whole blood. Coenzymes of redox reactions including oxidized/reduced nicotinamide adenine dinucleotide $\left(\mathrm{NAD}^{+}\right.$and $\left.\mathrm{NADH}\right)$ and nicotinamide adenine dinucleotide phosphate $\left(\mathrm{NADP}^{+}\right.$and $\left.\mathrm{NADPH}\right)$; the coenzymes of energy including adenosine triphosphate (ATP), adenosine diphosphate (ADP) and adenosine monophosphate (AMP) and the antioxidants including the sum of oxidized and reduced glutathione (GSSG and GSH) can be measured with essentially no additional effort along with the nearly 70 metabolites that were shown to be quantitated in serum/plasma, recently. Considering that coenzymes and antioxidants represent a sensitive measure of cellular functions in health and numerous diseases, the simple NMR spectroscopy method described here for profiling tissue and blood potentially opens a new avenue for app $\square$ ons in the metabolomics field.

Keywords: Redox and energy coenzymes; antioxidants; ${ }^{1} \mathrm{H}$ NMR; quantitation 


\section{Introduction}

- $\mathrm{NAD}^{+}, \mathrm{NADH}, \mathrm{NADP}^{+}$and NADPH mediate biochemical reactions fundamental to the functioning of living cells

- ATP along with ADP and AMP, represent the cellular energy currency.

- No simple method exists currently to measure all quantitatively and simultaneously

Conventional analysis method: Enzymatic Assay:

\section{Challenges:}

- Separate protocol required for each coenzyme.

- Interference from sample matrix

- Finite linear range

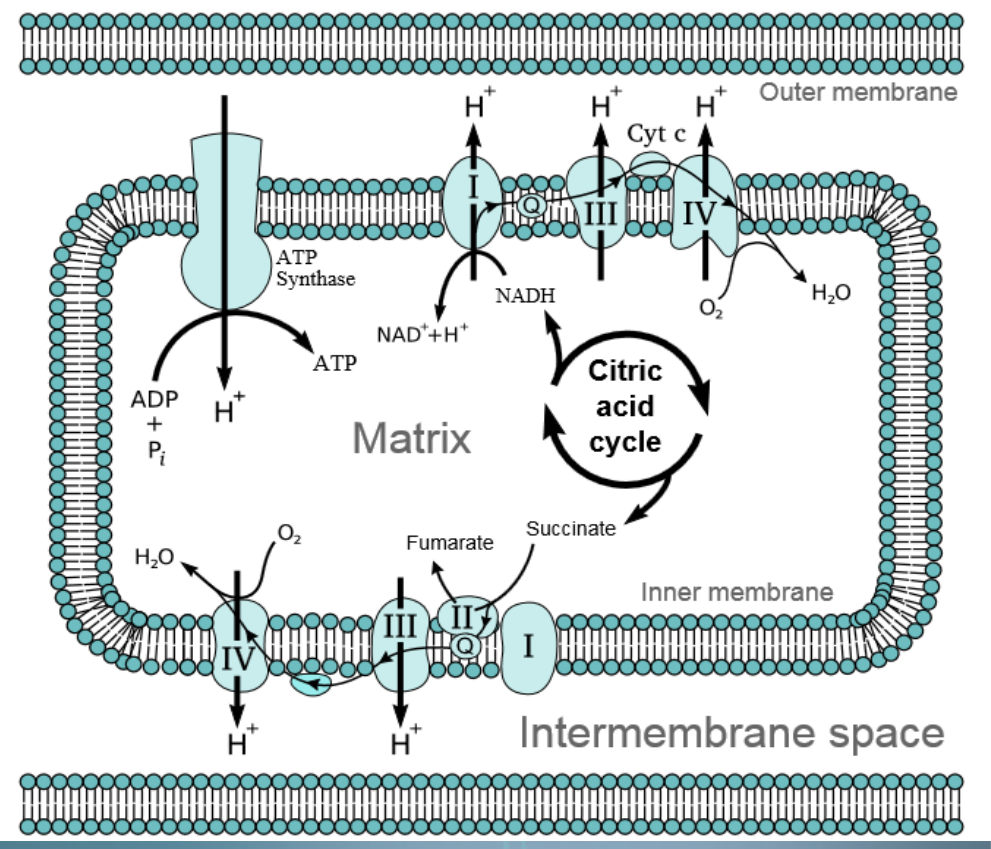


Yes, but...

\section{Challenges}

- Ion suppression

- Interference due to unit mass difference

- Interference due to similar chromatographic retention time

- In-source fragmentation
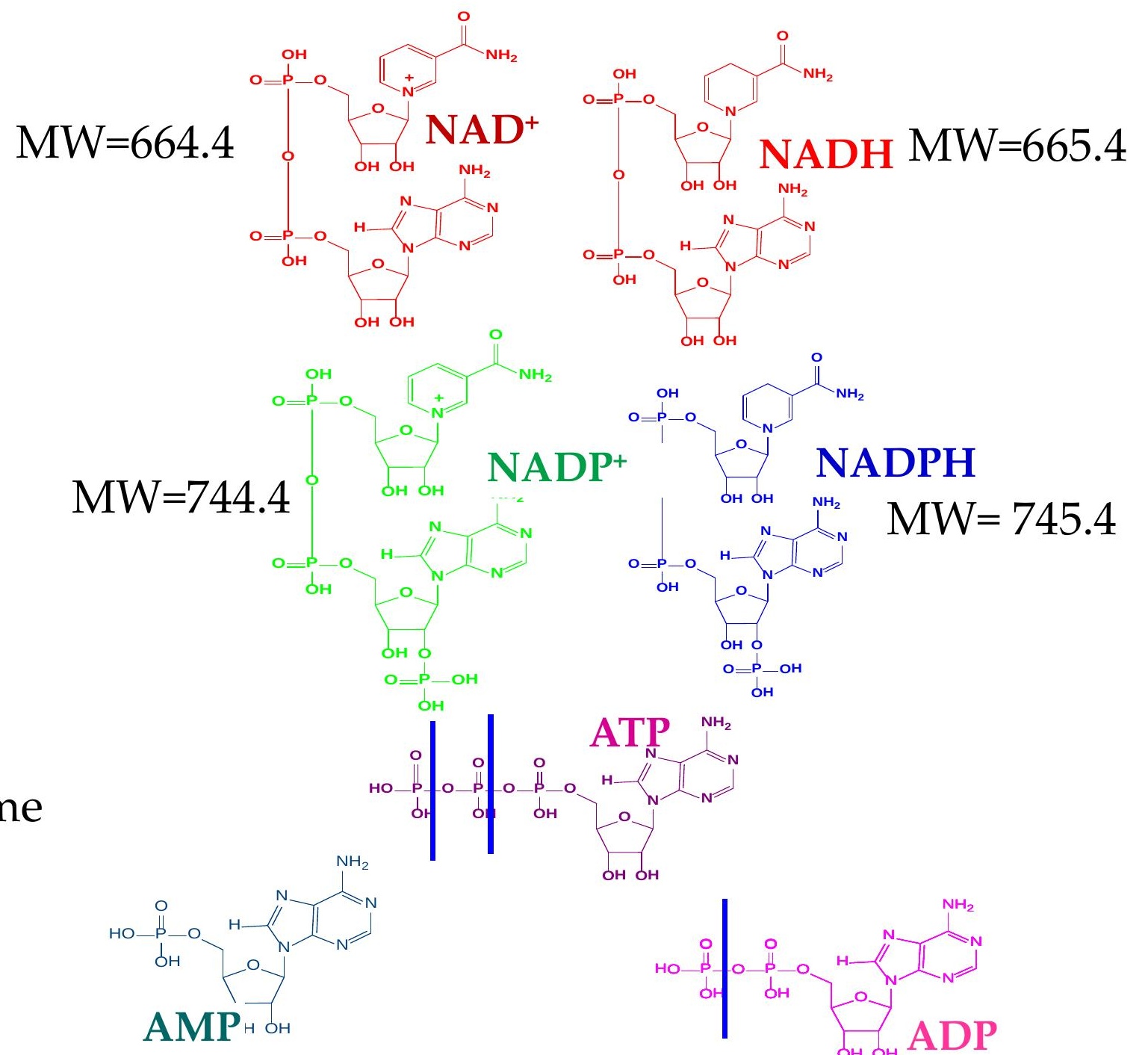
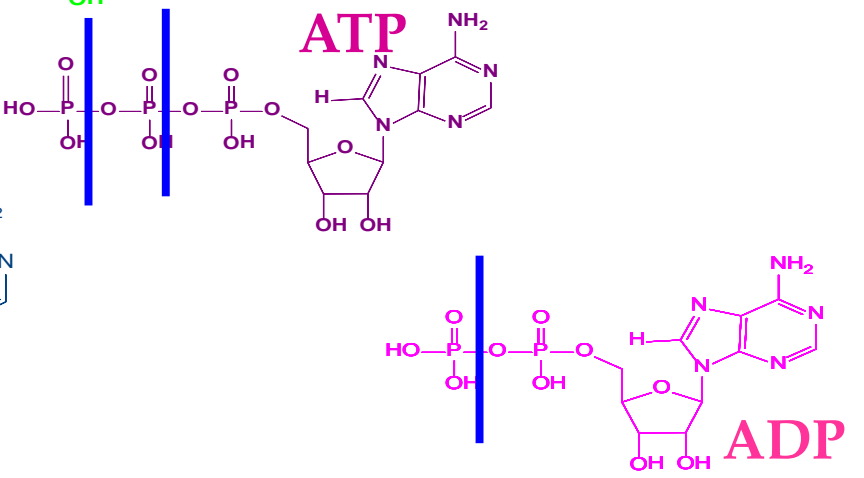


\section{Introduction}

\section{Challenges:}

- Complexity

- Similar structures

- Low concentration

- Instability

- Enzyme activity

- Oxidation
Can you Analyze by NMR Spectroscopy?
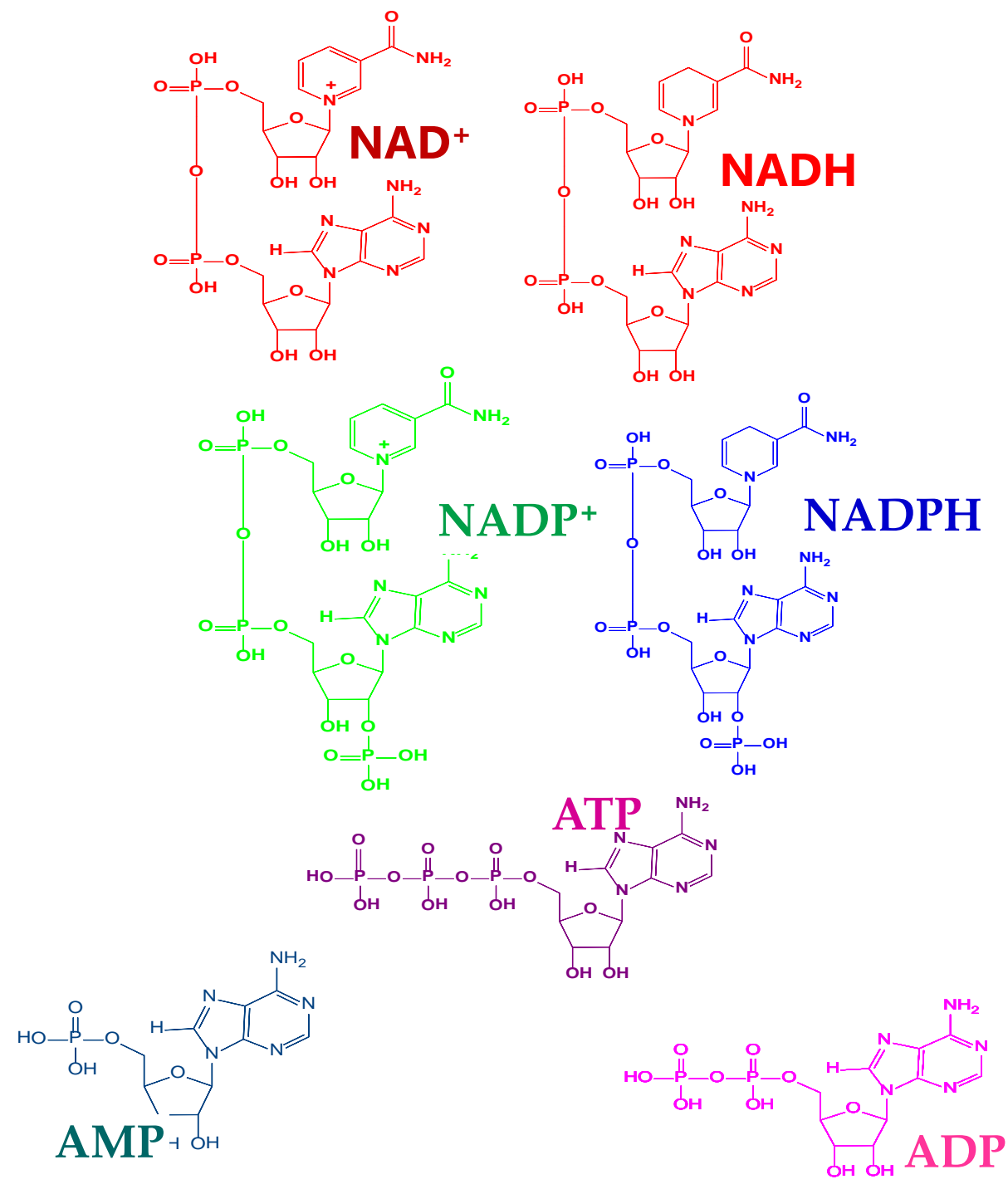


\section{Results and Discussion}

\section{Coenzymes in Tissue Extracts}
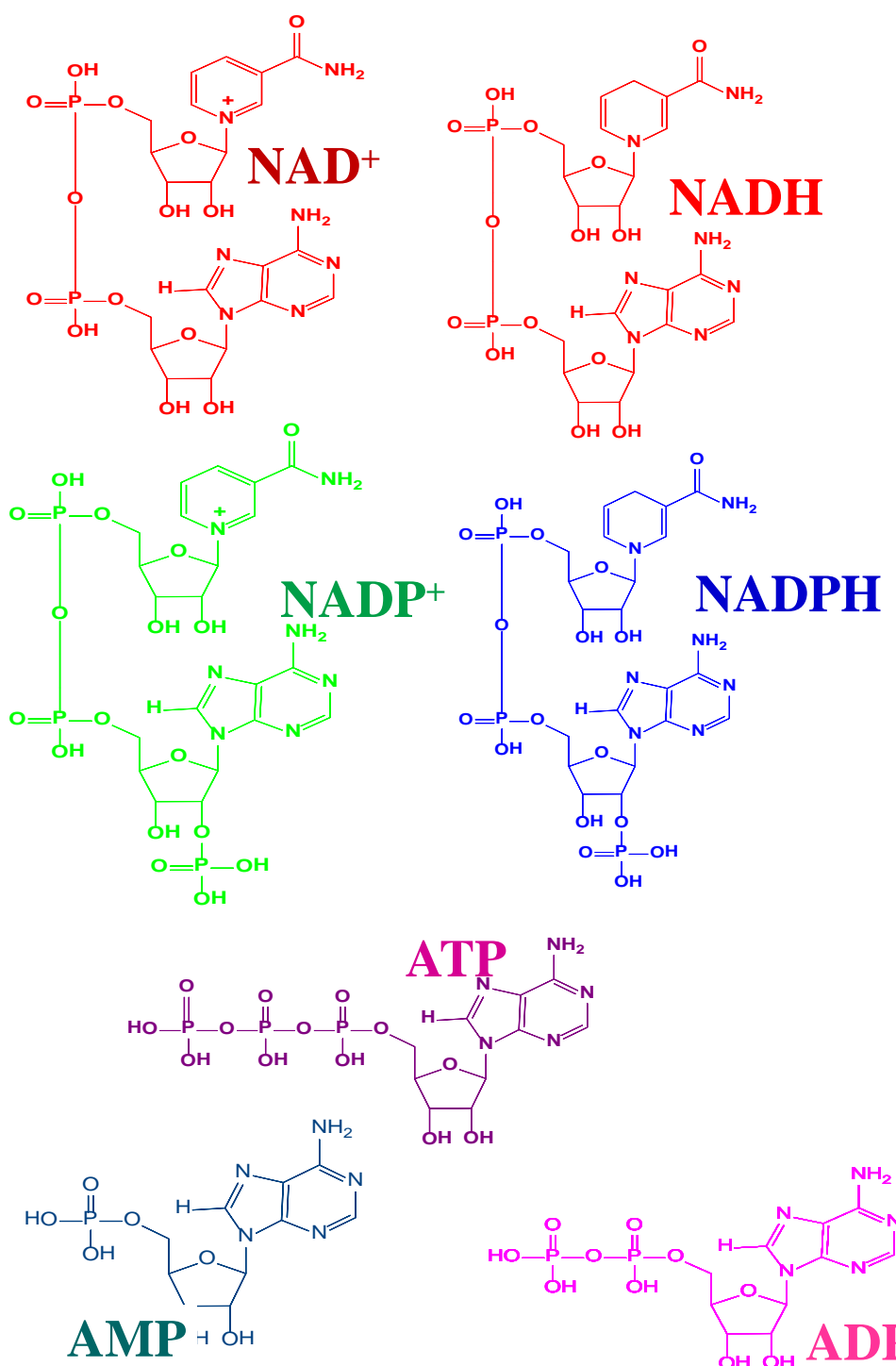

$\operatorname{ATP} N_{N}^{\mathrm{NH}_{2}}$
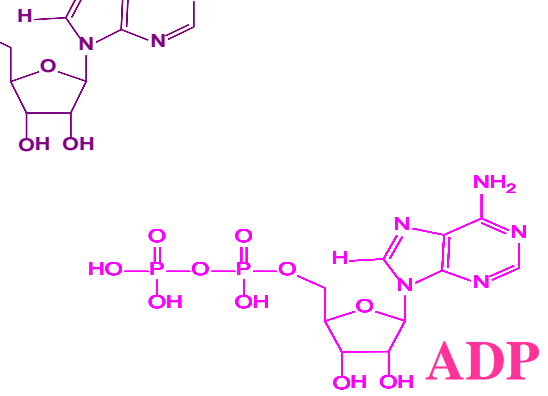

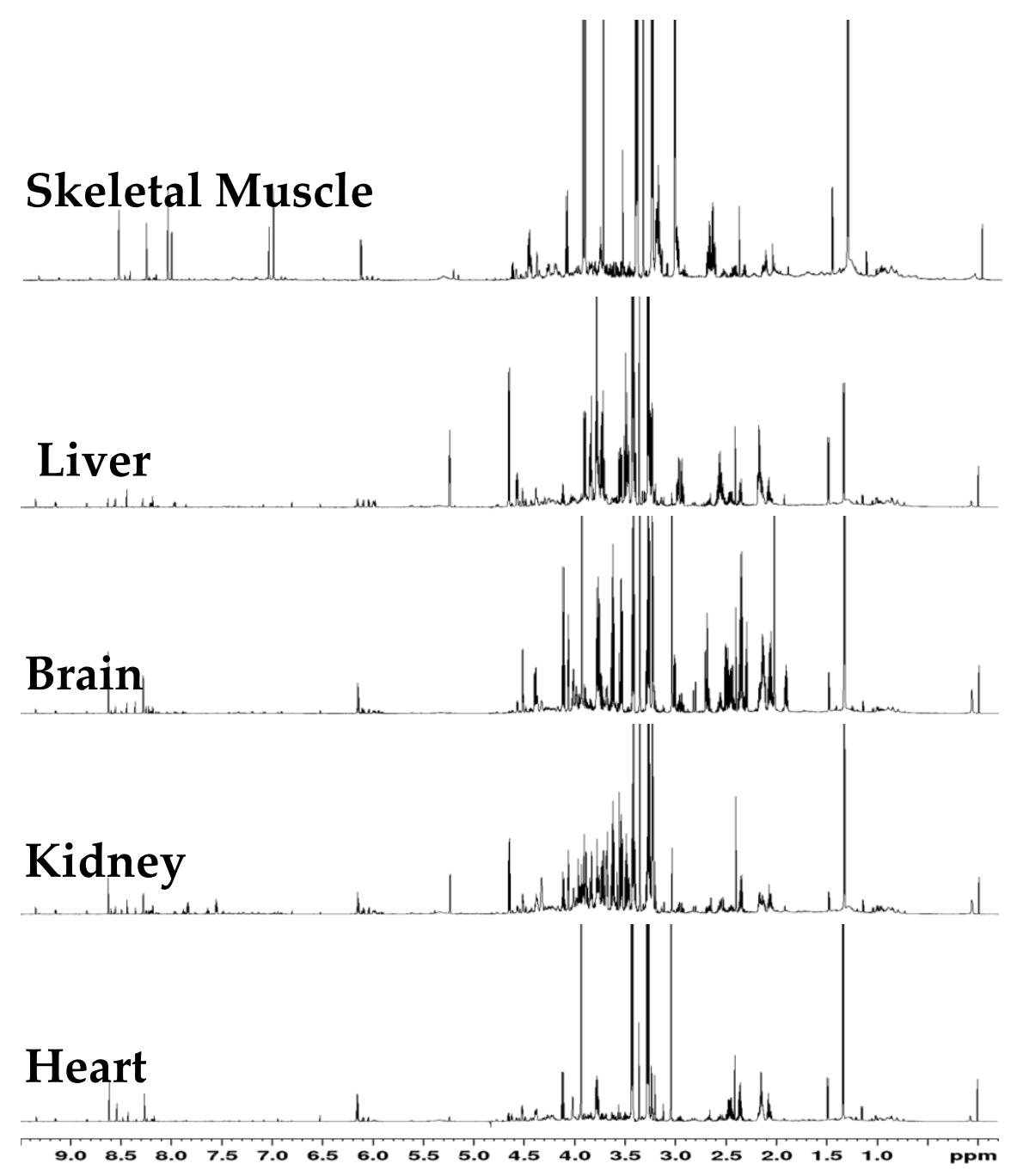




\section{Results and Discussion}

\section{Founding work for coenzymes analysis}

This is an open access article published under an ACS AuthorChoice License, which permits copying and redistribution of the article or any adaptations for non-commercial purposes.

\section{Expanding the Limits of Human Blood Metabolite Quantitation Using NMR Spectroscopy}

G. A. Nagana Gowda, ${ }^{\dagger} \dagger$ Yashas N. Gowda, ${ }^{\dagger}$ and Daniel Raftery $*, \dagger, \$, \S$

${ }^{\dagger}$ Northwest Metabolomics Research Center, Anesthesiology and Pain Medicine, and ${ }^{\ddagger}$ Department of Chemistry, University of Washington, Seattle, Washington 98109, United States

${ }^{\S}$ Fred Hutchinson Cancer Research Center, Seattle, Washington 98109, United States

Supporting Information

ABSTRACT: A current challenge in metabolomics is the reliable quantitation of many metabolites. Limited resolution and sensitivity combined with the challenges associated with unknown metabolite identification have restricted both the number and the quantitative accuracy of blood metabolites. Focused on alleviating this bottleneck in NMR-based metabolomics, investigations of pooled human serum combining an array of $1 \mathrm{D} / 2 \mathrm{D}$ NMR experiments at $800 \mathrm{MHz}$, database searches, and spiking with authentic compounds enabled the identification of 67 blood metabolites. Many of these $(\sim 1 / 3)$ are new compared with those reported previously as a part of the Human Serum Metabolome Database. In addition, considering both the high reproducibility and quantitative nature of NMR as well as the sensitivity of NMR chemical shifts to altered sample conditions, experimental protocols and comprehensive peak annotations are provided here as a guide for identification and quantitation of the new pool of blood metabolites for routine applications. Further,

Anal Chem. 2015 Jan 6;87(1):706-15

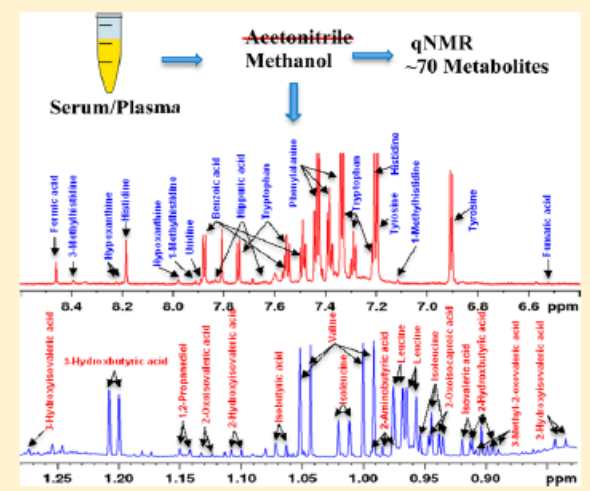

2nd International Electronic Conference on Metabolomics 20-27 November 2017 


\section{Results and Discussion}

Founding work for coenzymes analysis

Nearly 70 metabolites identified and quantitated in human blood

$\sim 35 \%$ were newly identified

Unprecedented for 1D NMR.

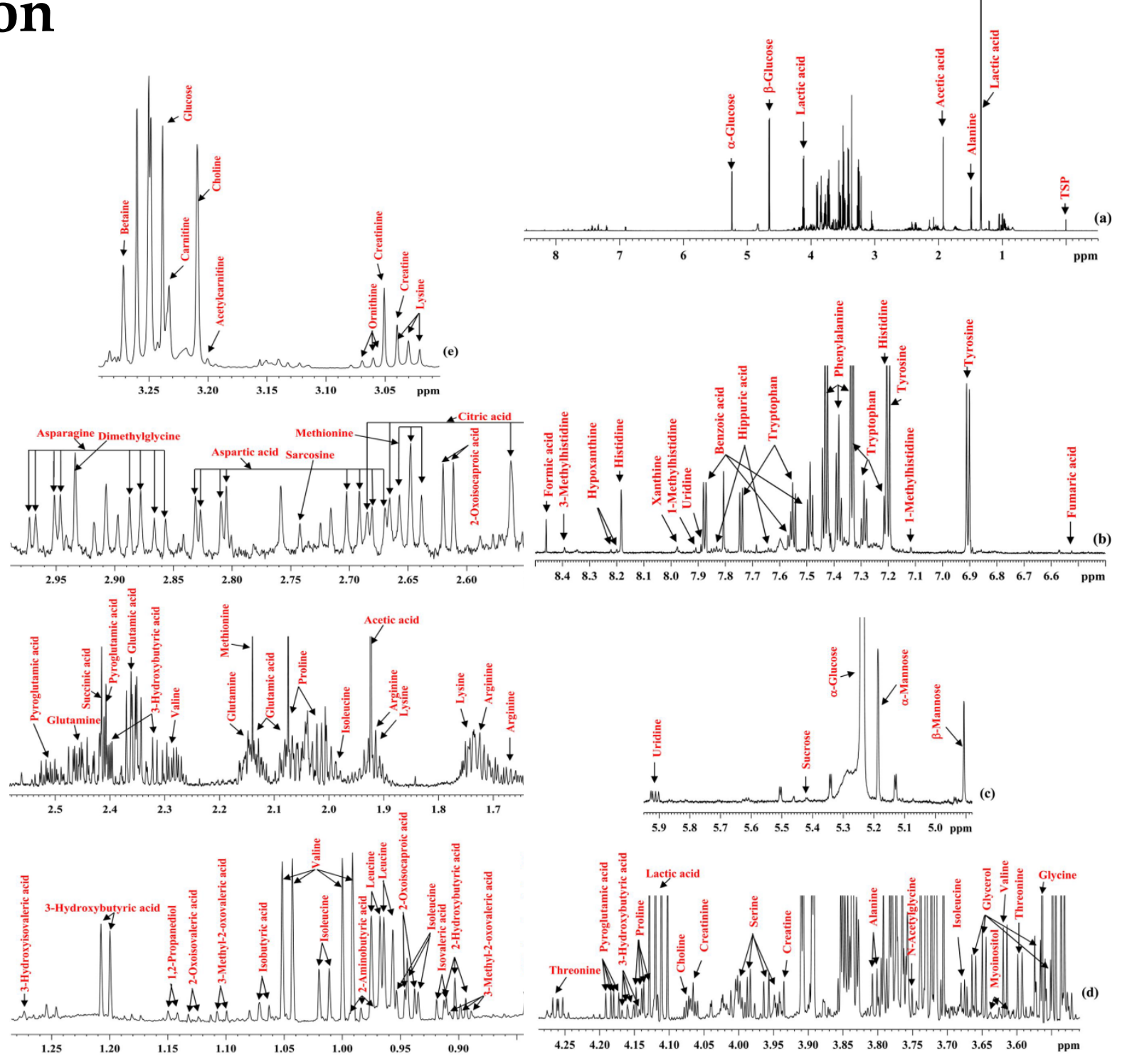

Anal Chem. 2015 Jan 6;87(1):706-15 


\section{Results and Discussion}




\section{Results and Discussion:}

\section{Coenzymes in Different Tissues}
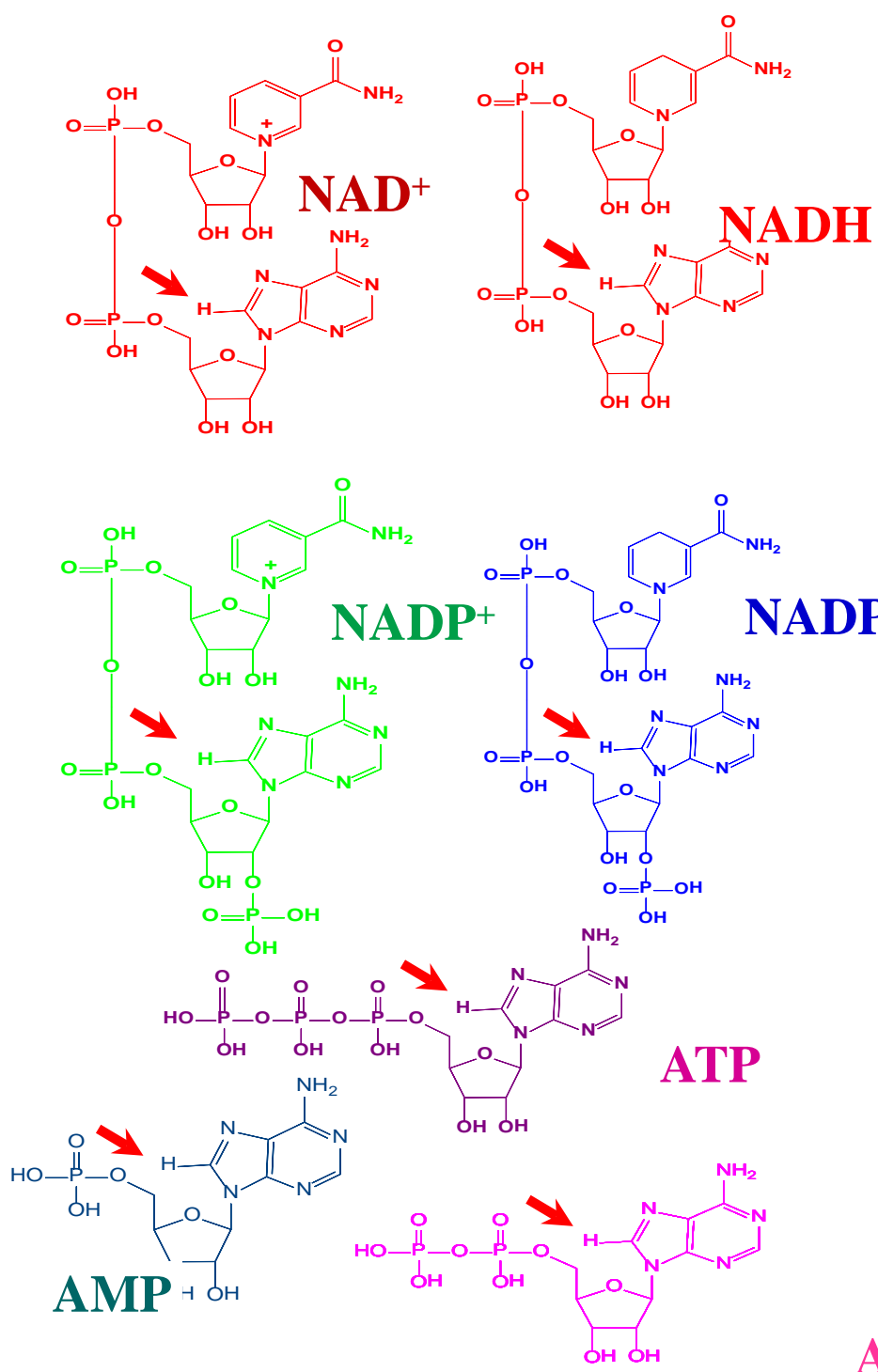

NADPH

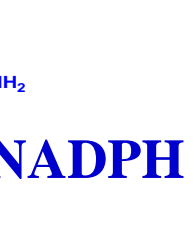

$\mathrm{OH} \mathrm{OH}$

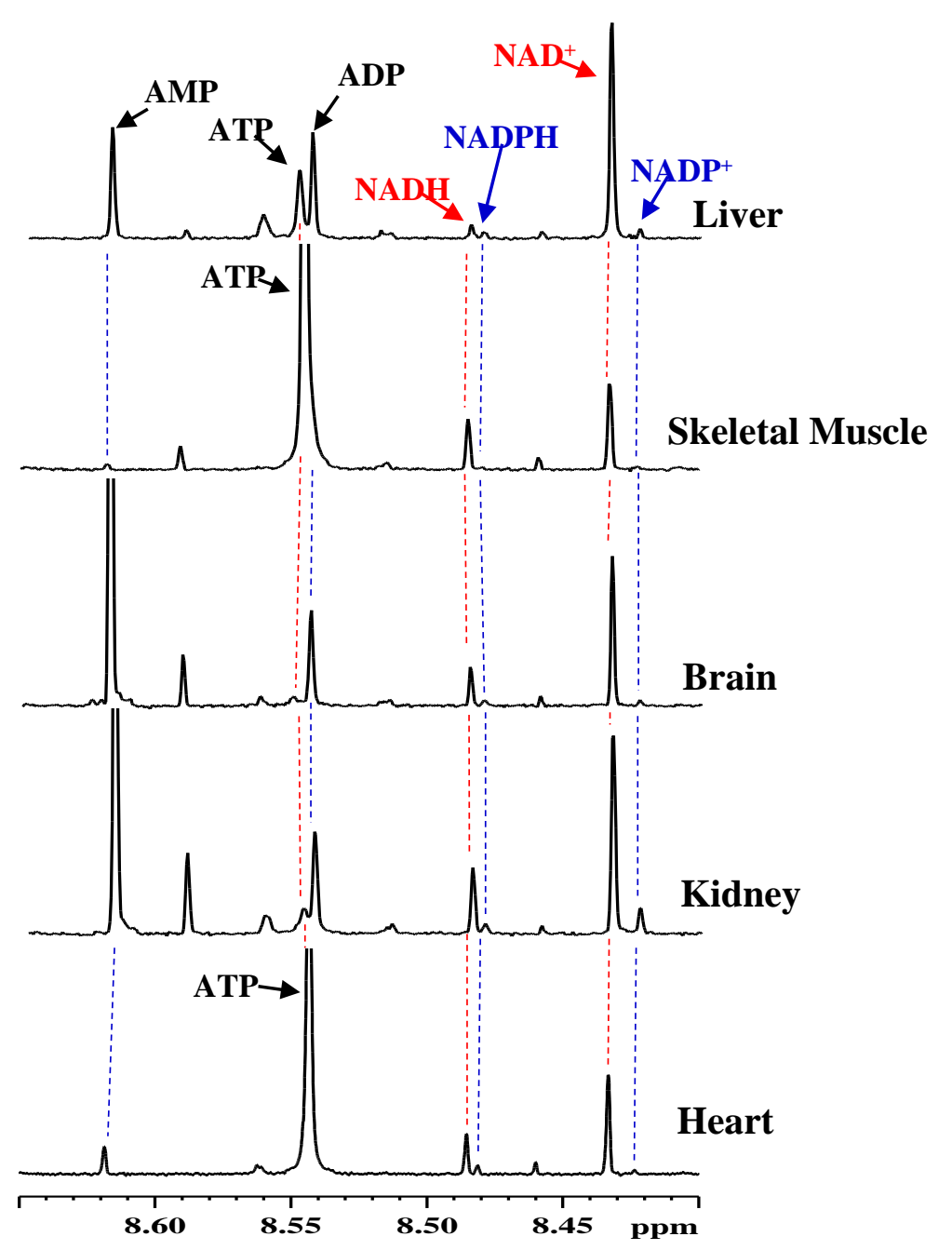

ADP 2nd International Electronic Conference on Metabolomics

20-27 November 2017 


\section{Results and Discussion}

Sensitivity of coenzymes to tissue harvesting/extraction

The same mouse heart tissue extracted under different conditions

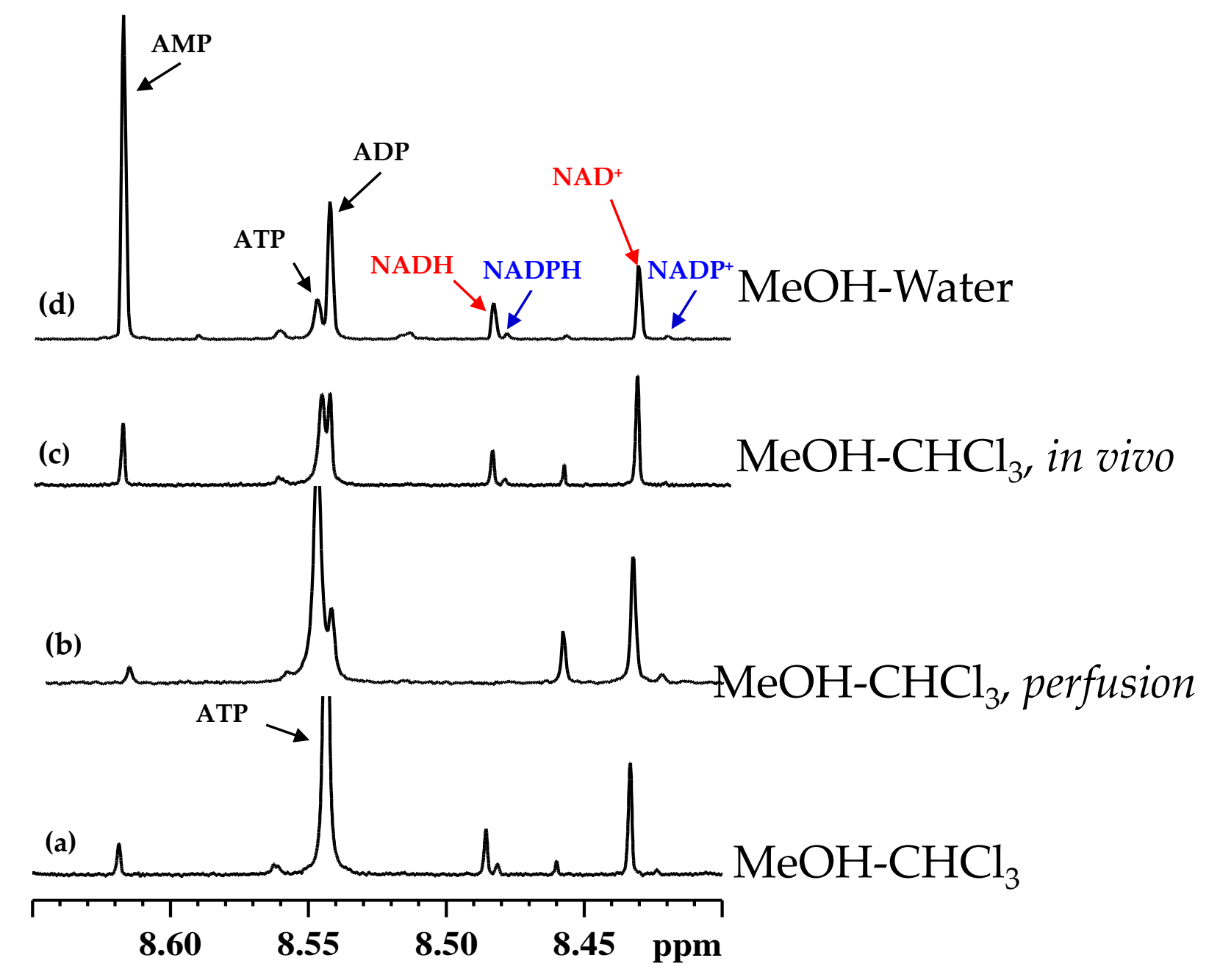




\section{Results and Discussion}

\section{Simultaneous Analysis of Coenzymes}
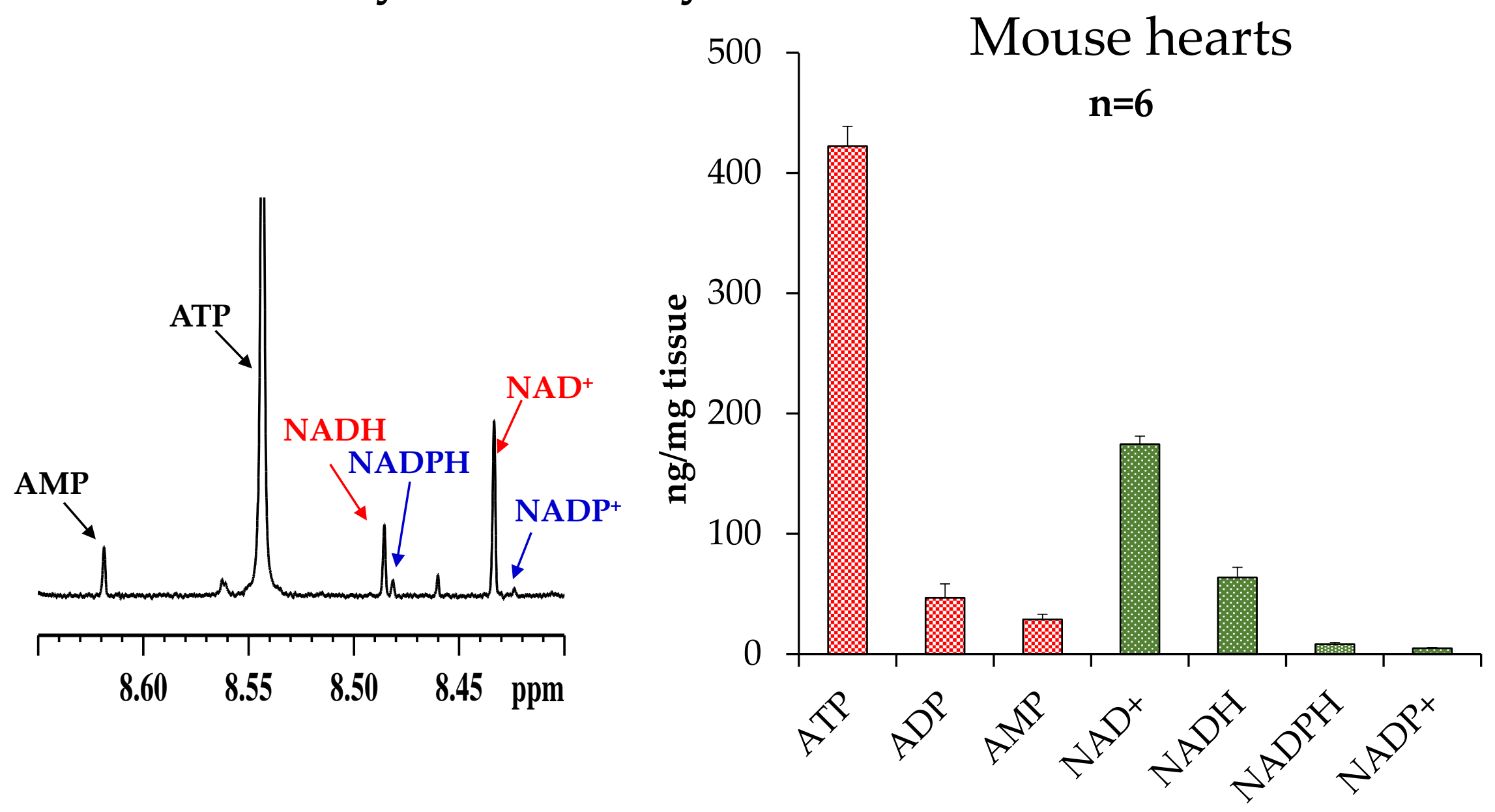


\section{Results and Discussion}

\section{Coenzymes from $\sim 5 \mathrm{mg}$ tissue}
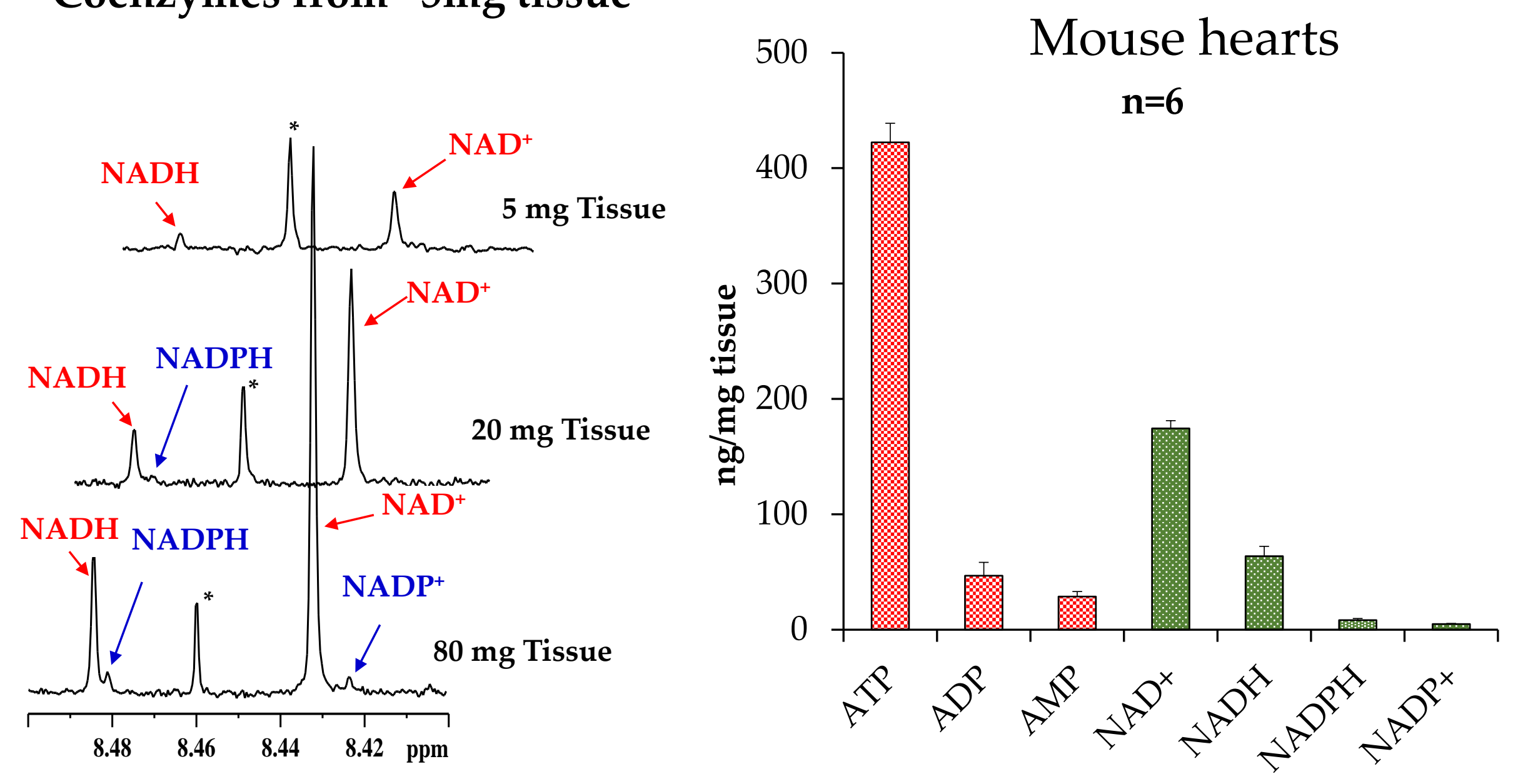


\section{Results and Discussion}

\section{Coenzymes and Antioxidants in Whole Blood}

- Blood Metabolomics: Serum or Plasma

- Whole blood: serum/plasma + Cells

-Nearly $50 \%$ of blood volume is cells and $99 \%$ of cells are RBC.

- Whole Blood Metabolomics:

-Provides access to wider pool of metabolites with no additional efforts. 


\section{Results and Discussion Coenzymes and Antioxidants in Blood}

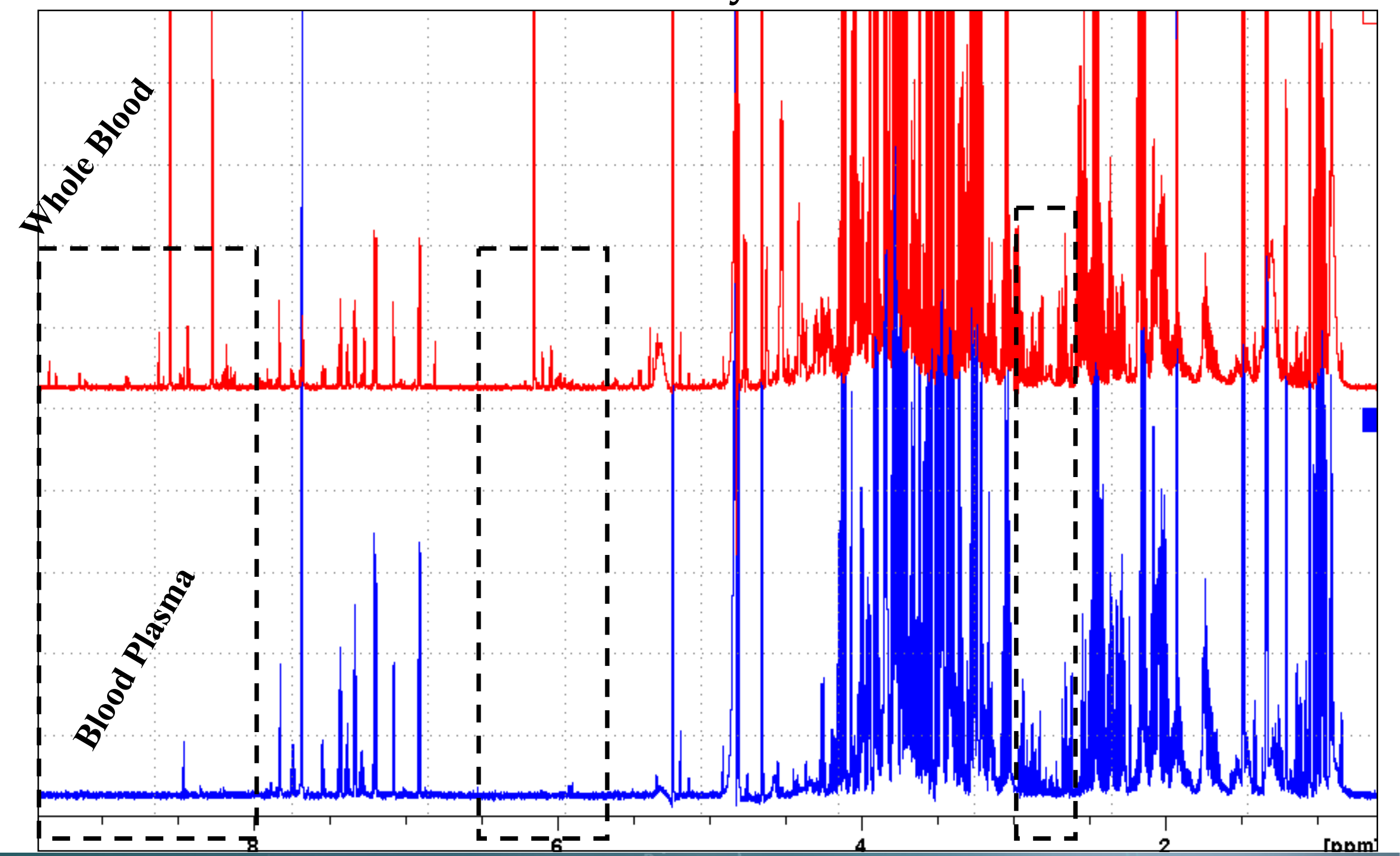

2nd International Electronic Conference 


\section{Results and Discussion}

\section{Coenzymes in Whole Blood extract}

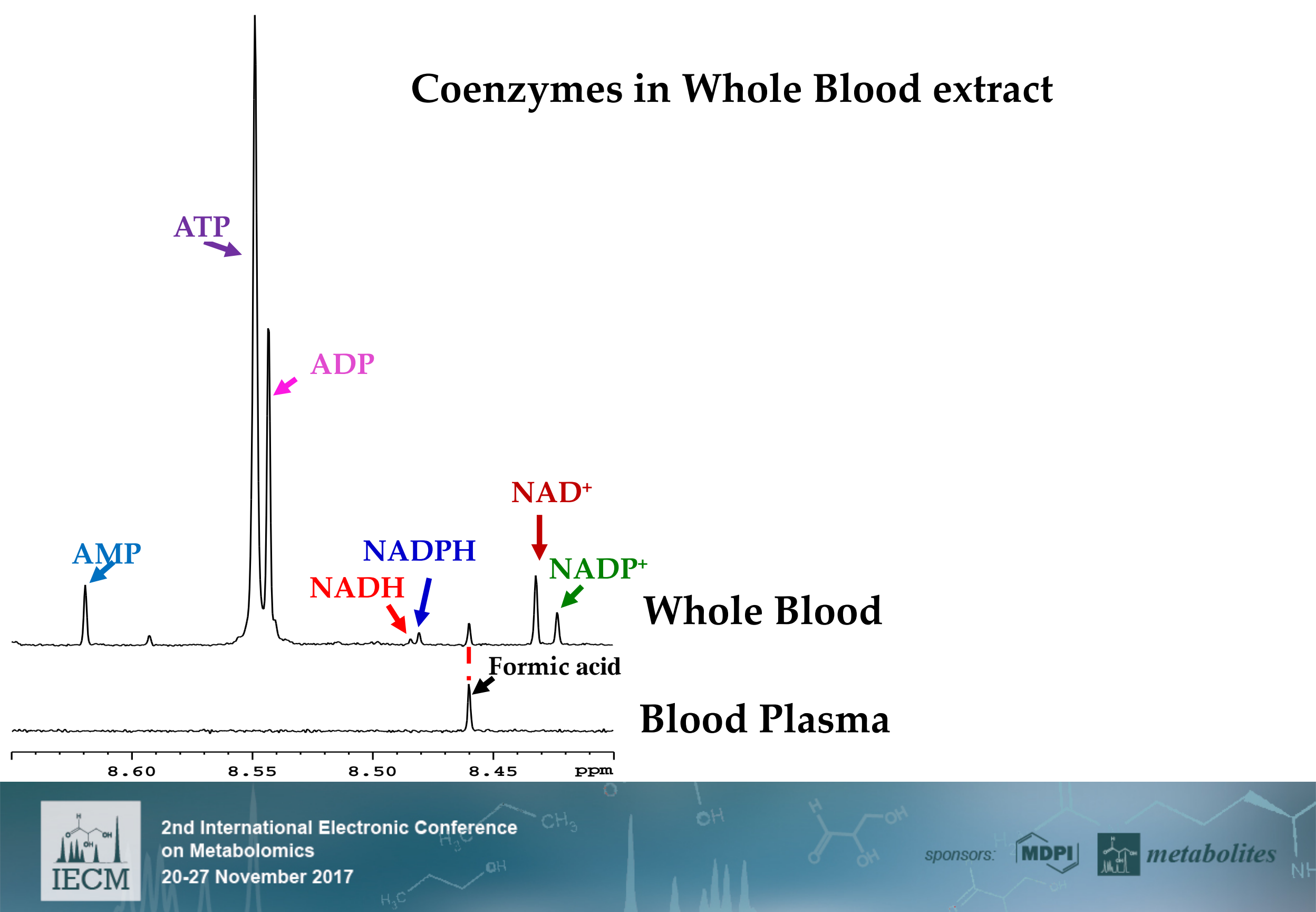




\section{Results and Discussion}

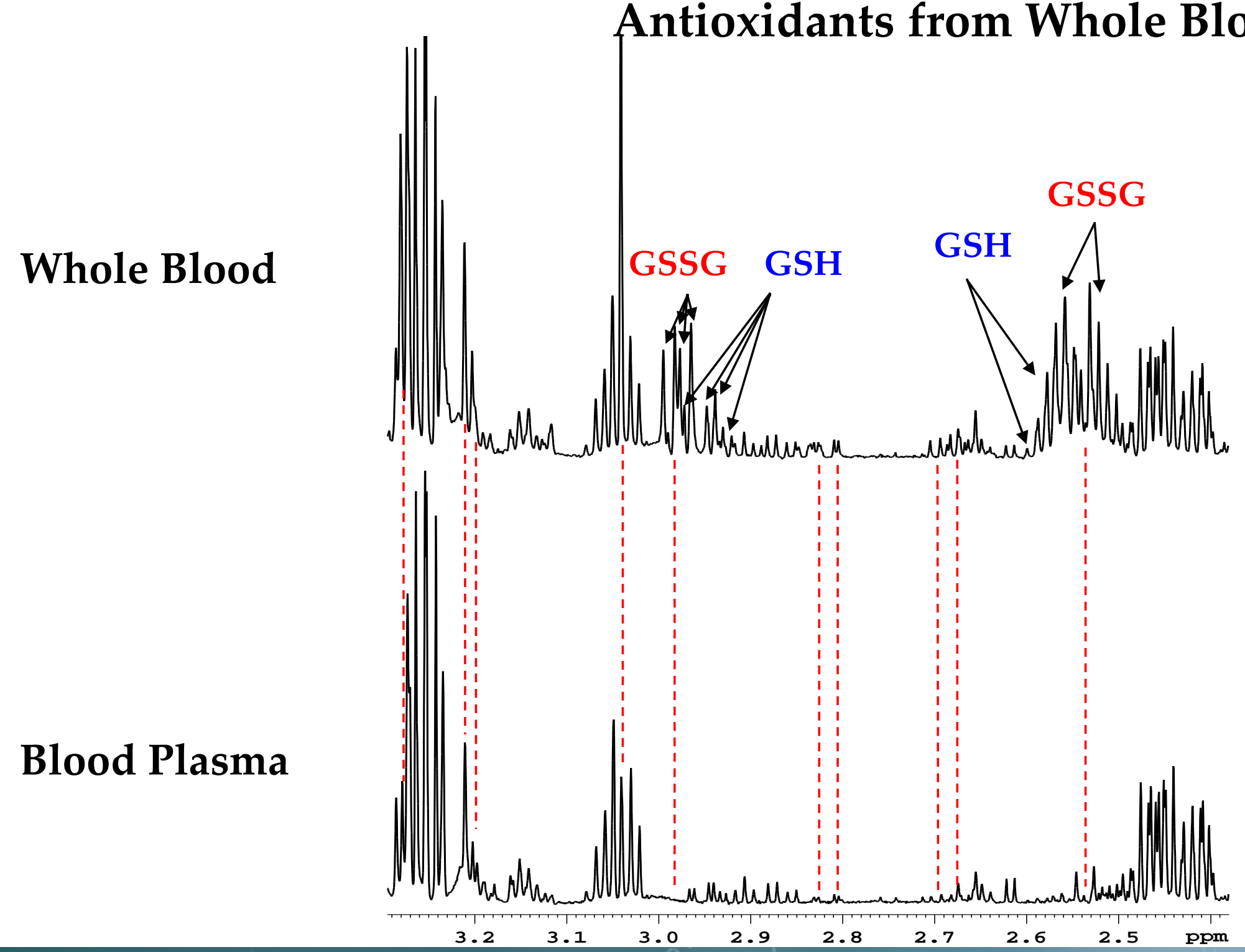




\section{Results and Discussion}

\section{Peak Ratios are Same as Coenzyme Ratios}

NAD + NADH

$\mathrm{NADP}^{+} / \mathrm{NADPH}$

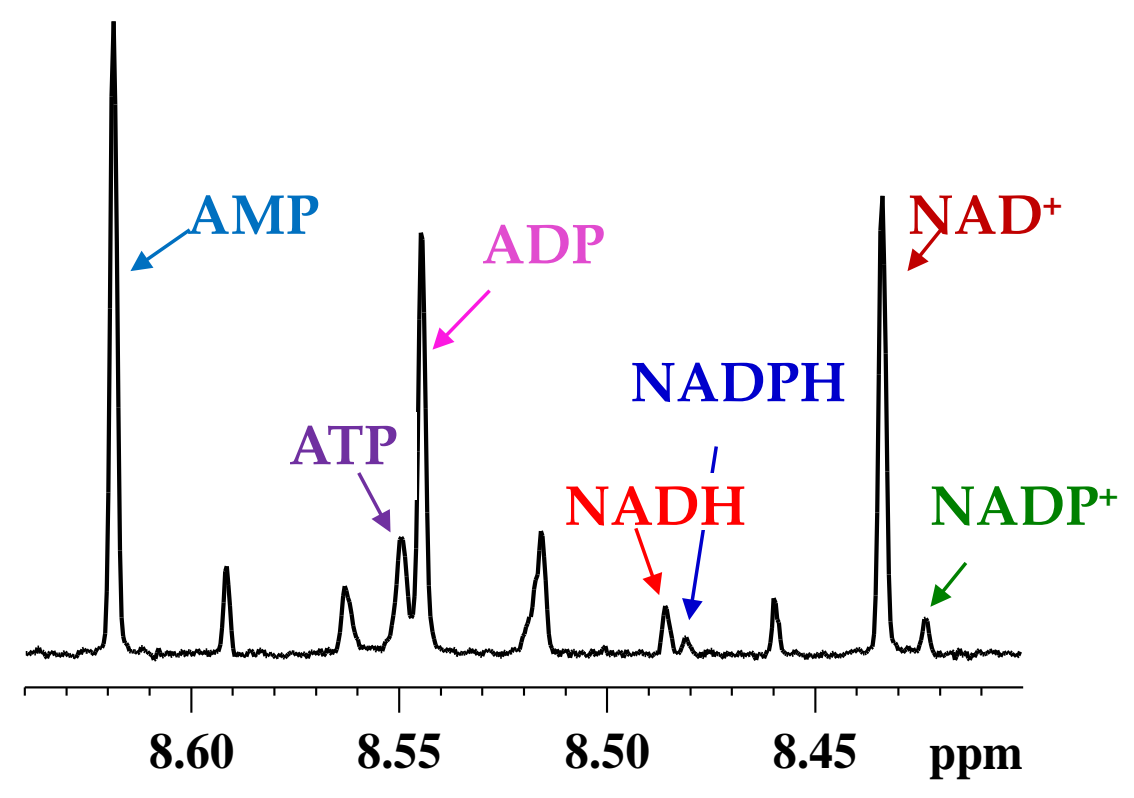

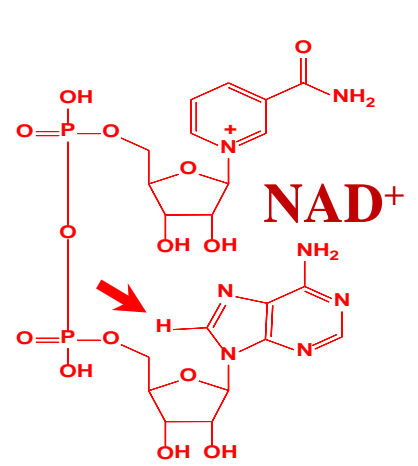
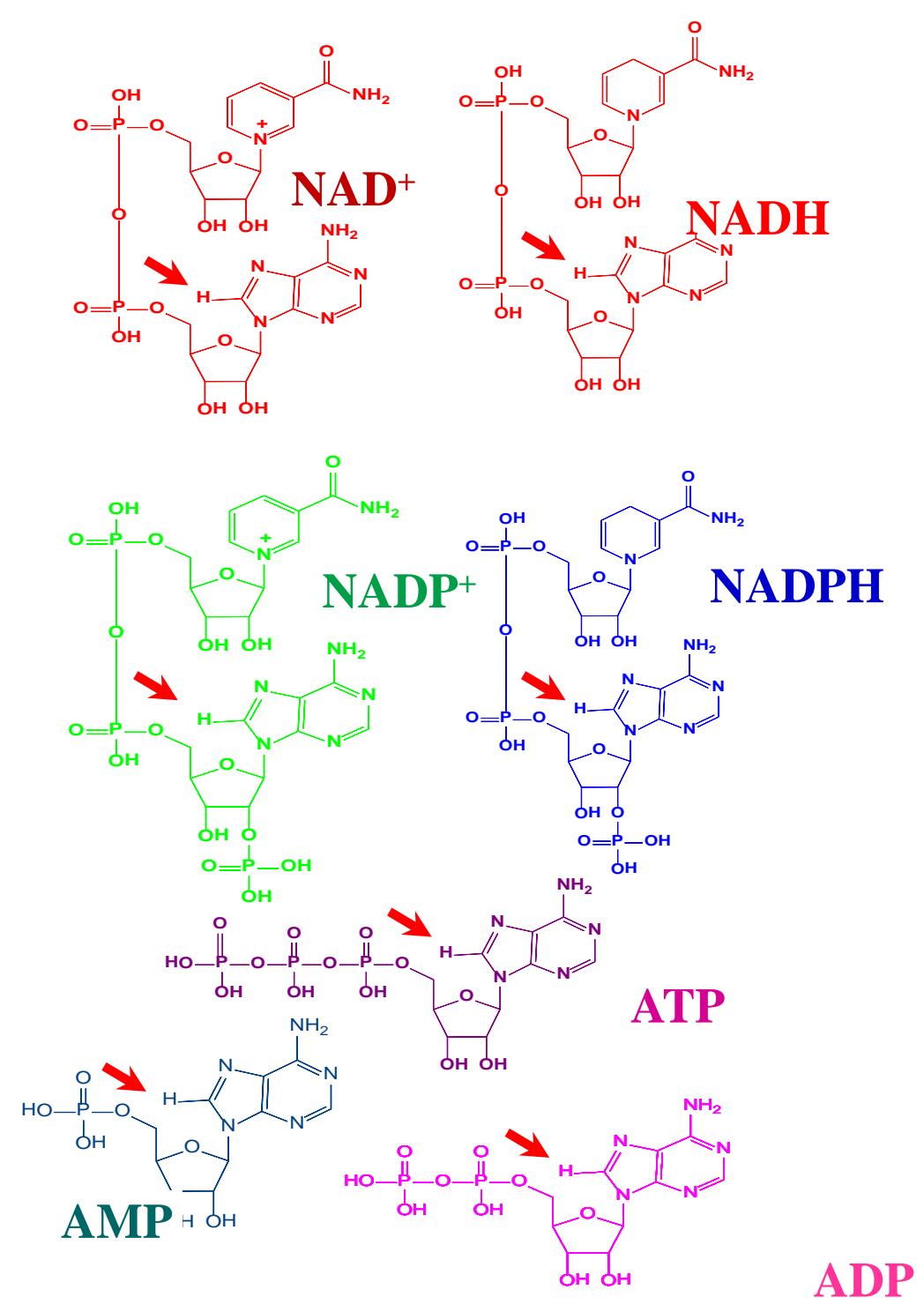

2nd International Electronic Conference on Metabolomics

20-27 November 2017 


\section{Results and Discussion}

\section{Biological validation}

Mice with Ndufs4 gene Knockout

- Ndufs4 protein critical for mitochondrial function and ATP synthesis.

- Ndufs4-KO: NADH/NAD ${ }^{+}$ratio
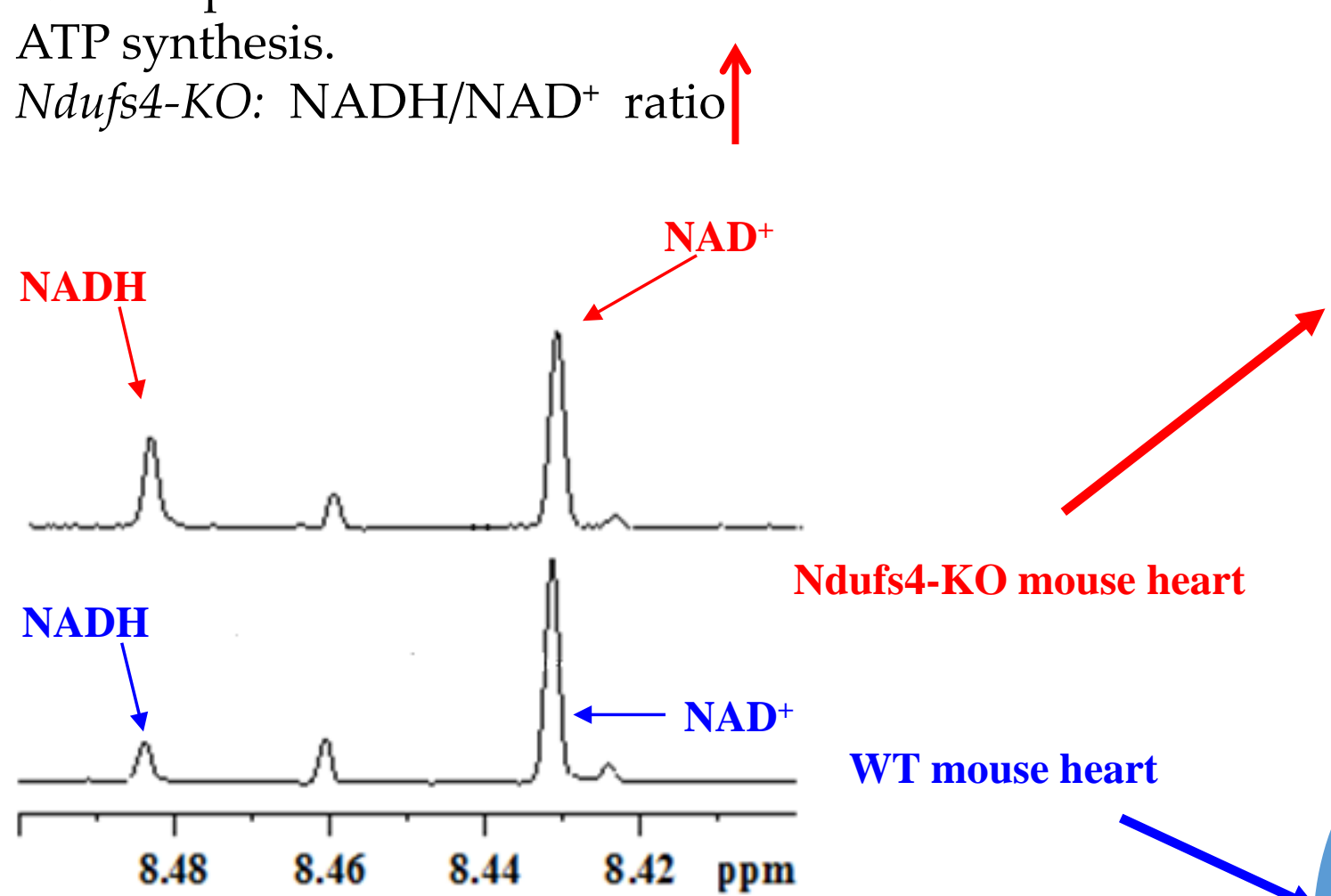

Ndufs4-KO mouse heart

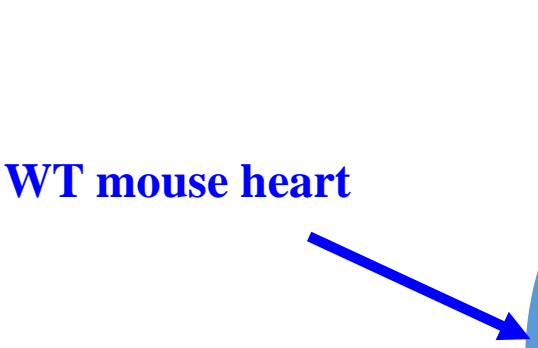

Ndufs4-KO mouse heart

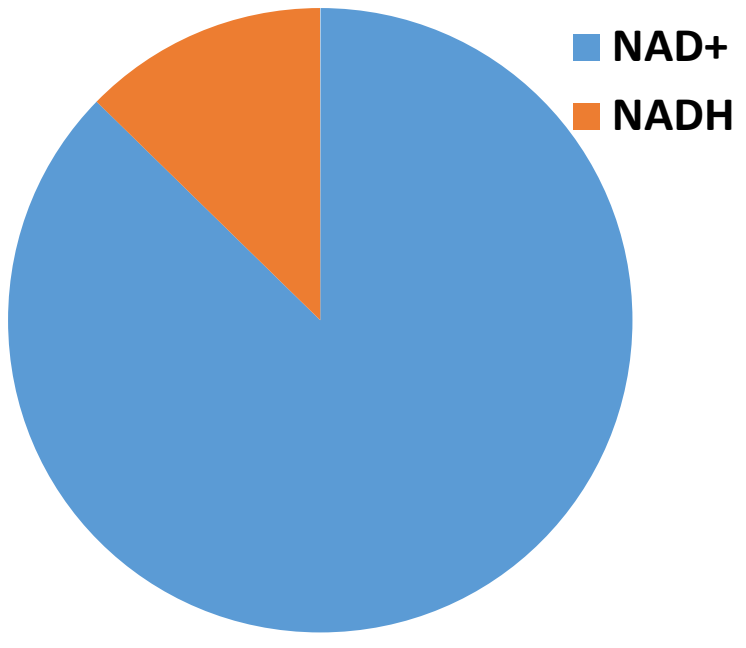

WT mouse heart 


\section{Results and Discussion}

\section{Overexpression of NAMPT Protein}

(Nicotinamide phosphoribosyltransferase)

- Increased $\mathrm{NAD}^{+}$synthesis

- $\mathrm{NAD}^{+/} \mathrm{NADH}$ pool $\uparrow$

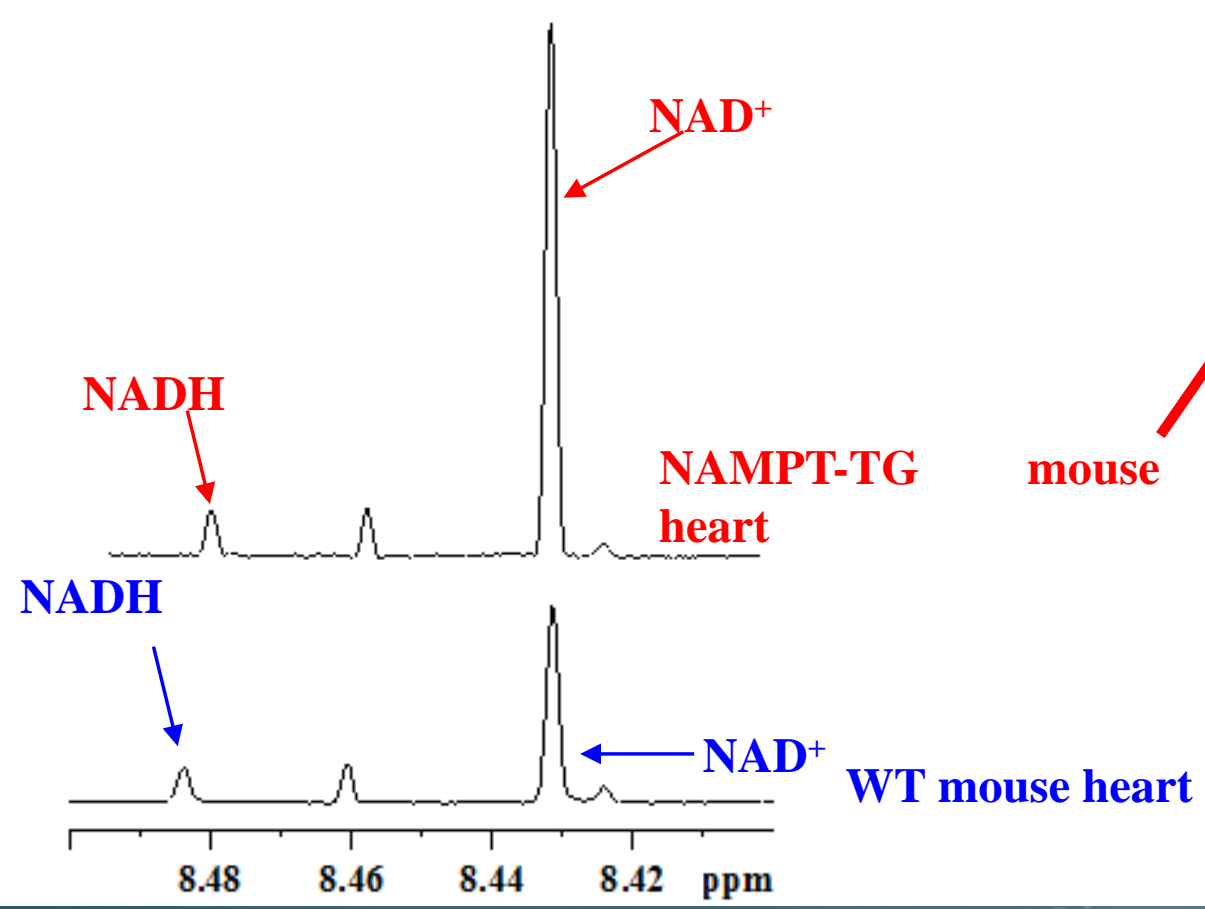




\section{Both NAMPT-TG/Ndufs4-KO}

- $\quad$ Ndufs4-KO: NADH $\uparrow$

- $\quad$ NAMPT-TG : NADH/NAD ${ }^{+}$pool $\uparrow$

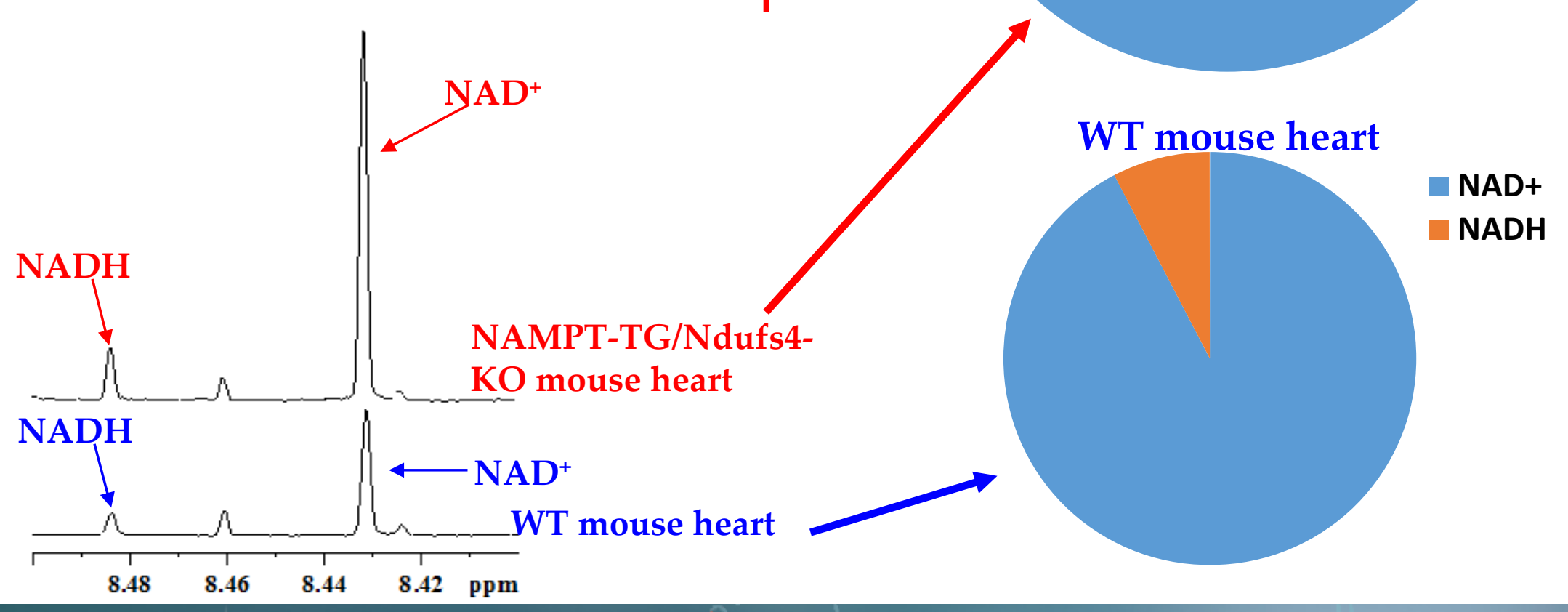




\section{Results and Discussion}

\section{Degassing Halts NADH/NADPH Oxidation}

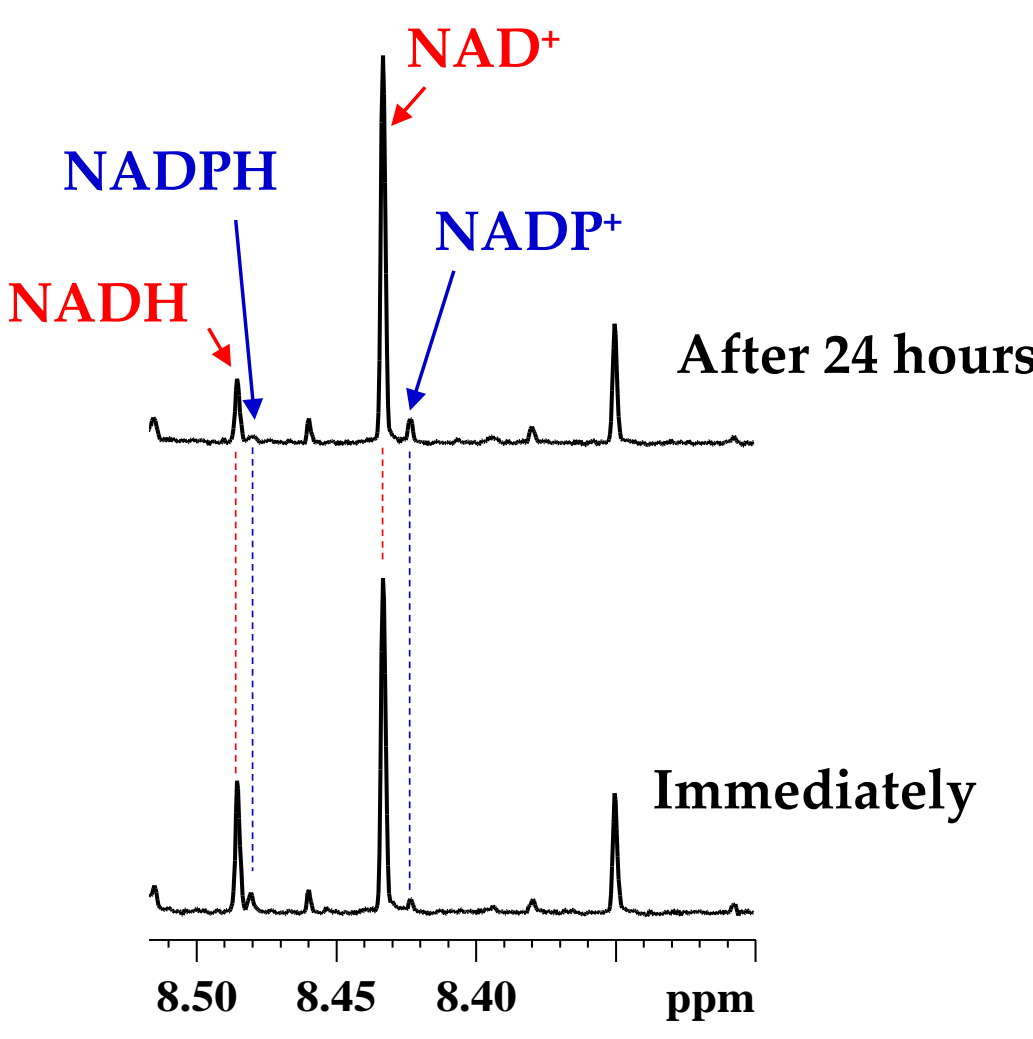

No degassing

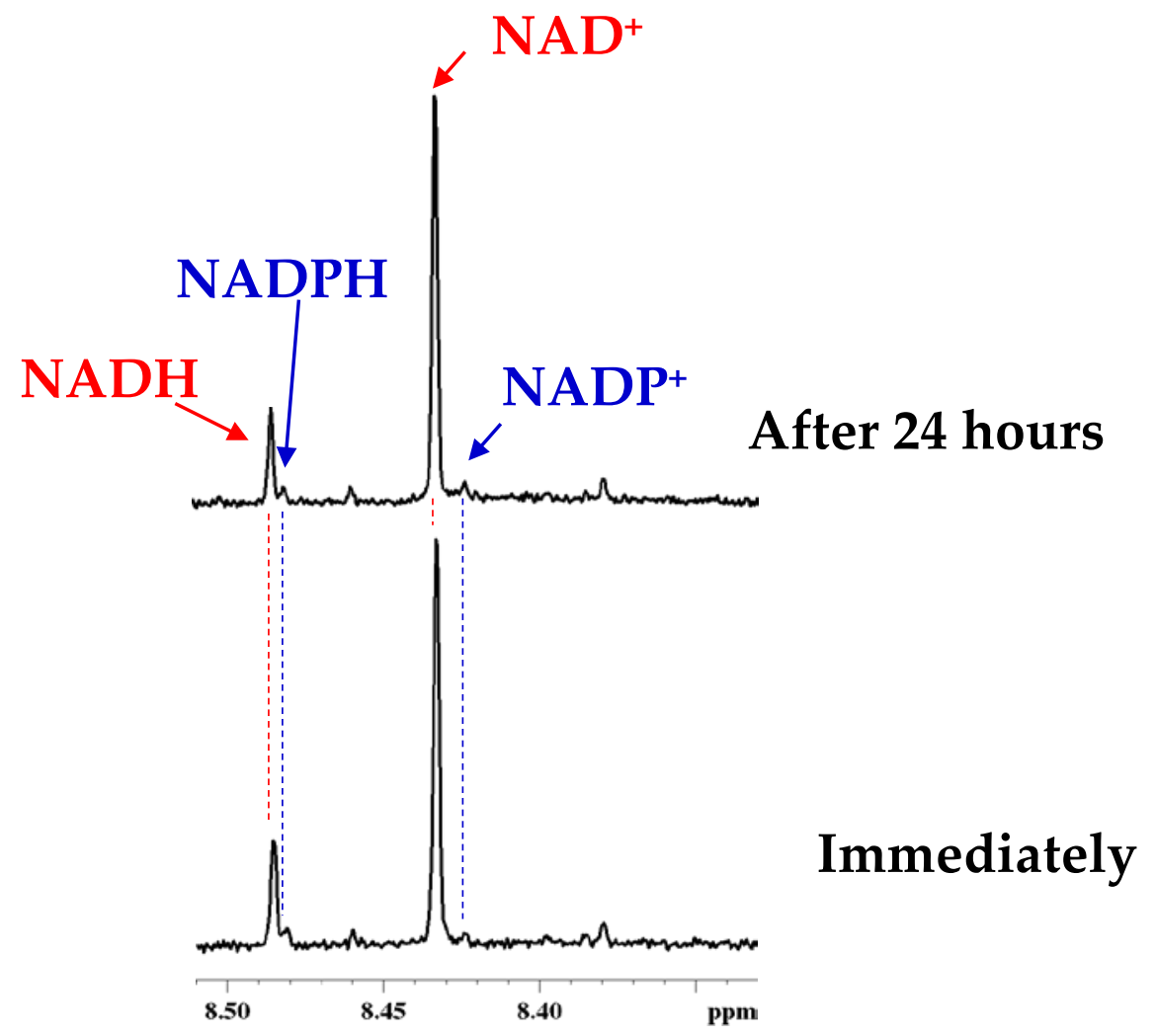

Degassing 


\section{Results and Discussion}

\section{Much More Than the Coenzymes}

Antioxidants

\section{TCA Cycle}

Glycolysis

\section{Amino acids}

Carboxylic acids

Creatine/Phosphocreatine
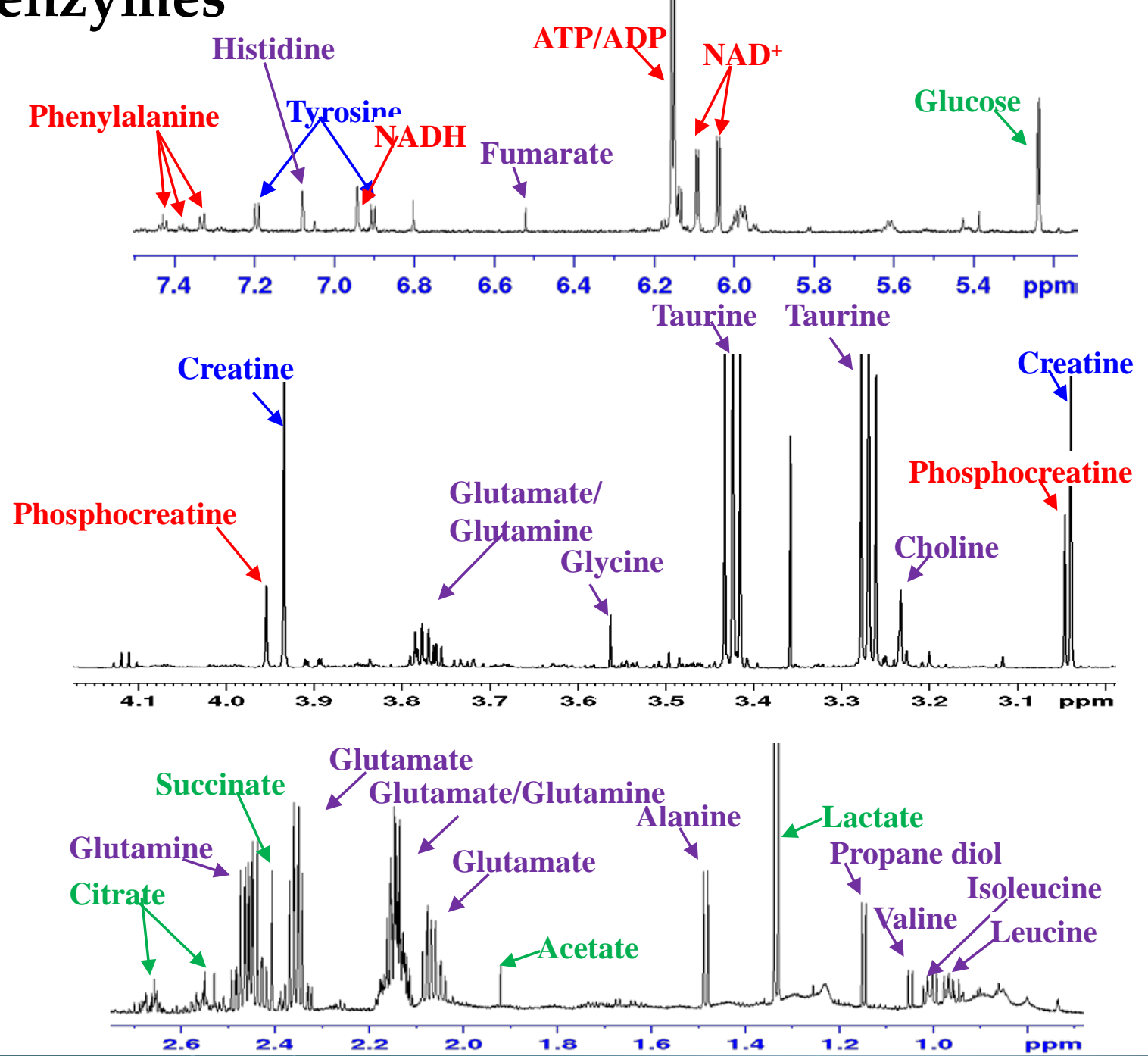


\section{Results and Discussion}

Concentrations of coenzymes, antioxidants and other metabolites in human blood
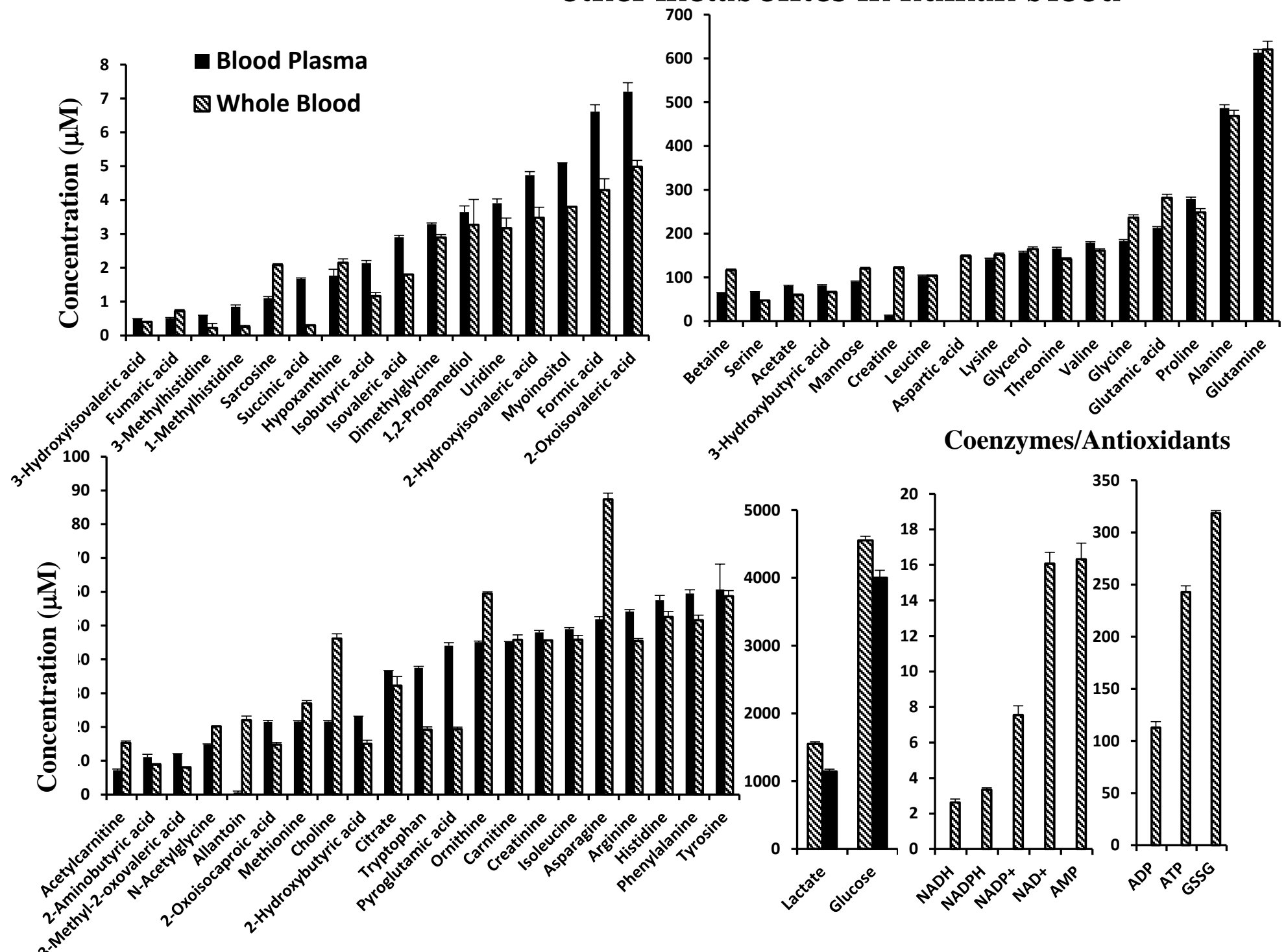


\section{Conclusions}

- We provide a simple NMR method for simultaneous quantification of coenzymes of cellular redox reactions and energy in tissue and blood.

- We demonstrate importance of harvesting/extraction methods for reliable analysis, as many coenzymes can evade detection wholly or partly depending on the method.

- We show that over a period of time NADH and NADPH levels diminish due to oxidation in solution; however, degassing can halt such a change.

- Most of the coenzymes can be measured using as little as $5 \mathrm{mg}$ tissue and a further improvement in the detection limit can be achieved using sensitivity enhancement methods such as using micro-coil probes.

- The ability to measure coenzymes and antioxidants along with a vast pool of other metabolites using NMR potentially opens a new chapter in the metabolomics field. 


\section{Supplementary Materials}

Links:

http://pubs.acs.org/doi/abs/10.1021/acs.analchem.7b00171

http://pubs.acs.org/doi/abs/10.1021/acs.analchem.6b00442

http://pubs.acs.org/doi/abs/10.1021/ac503651e 


\section{Acknowledgments}

\section{Funding:}

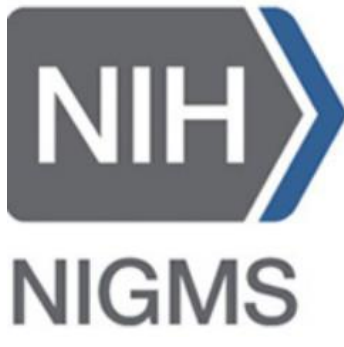

W

UNIVERSITY of WASHINGTON

Royalty Research Fund (RRF)

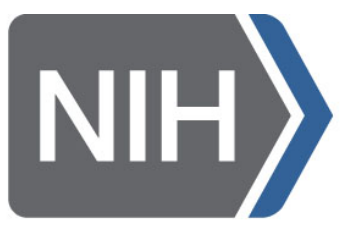

National Institute of Biomedical Imaging and Bioengineering

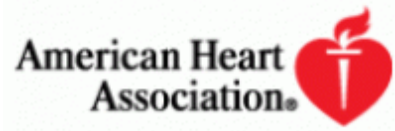

Learn and Live.

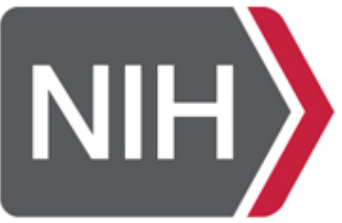

National Heart, Lung, and Blood Institute 